\title{
A Crucial Investigation of Facial Skin Colour Research Trend and Direction
}

\author{
Mohammed Hazim Alkawaz ${ }^{1,2}$, Dzulkifli Mohamad ${ }^{1}$, Ahmad Hoirul Basori ${ }^{3}$ and Farhan \\ Mohamed $^{1}$ \\ ${ }^{1}$ Faculty of Computing, Universiti Teknologi Malaysia, Malaysia \\ ${ }^{2}$ Faculty of Computer Sciences and Mathematics, University of Mosul, Mosul, \\ Iraq \\ ${ }^{3}$ Interactive Media and Human Interface Lab., Department of Informatics, Faculty \\ of Information Technology, Institut Teknologi Sepuluh Nopember Surabaya, Indo- \\ nesia \\ hamohammed5@live.utm.my,dzulkifli@utm.my,hoirul@its-sby.edu,far- \\ han@utm.my
}

\begin{abstract}
The research on facial animation is growing and became more sensible in terms of three dimensional data. The emergence of laser scans and advanced 3D tools imparted further towards the rapid development of complex facial model. This paper has given an overview on facial animation techniques such as blend shape interpolation, facial action coding system and parameterization. The major challenges, excitement, applications, recent progress and the future of various diversified facial animation methods are highlighted as well. The emotional theory, involving the cognition as one of the elements of emotion and emphasizing on somatic factors describing emotional expressions and its perception as the other elements are presented. The essential factors responsible for emotional colors manifestations to an individual are the key issues. The notable theories and models of Goethe, Claudia Cortes, Naz Kaya, Color Wheel Pro, Shirley Willet and Yau $X$ ueon facial colors expressions are critically commented and a facile comparison is carried out. Furthermore, a detailed discussions on facial colors appearance are provided using image based skin color analysis and synthesis, reflectance based model, bidirectional texture function, physiology measurement based and emotional appearance models. The paper completed with the remarkable features official expression comprised of facial action coding system and MPEG-4 facial animation that enable considerable improvement on facial animation technology. Our in-depth appraisal on facial animation may contribute as taxonomy towards the development of human facial skin colors on $3 D$ avatar including facial action coding system, emotion theory, color theory, animation via blending and image based color analysis useful for various applications.
\end{abstract}

Keywords: Animation, Computer Graphics, Virtual Reality, Avatars, Hemoglobin and Melanin

\section{Introduction}

Recent development in digital technology for stabilizing the high-speed processing of gigantic information is overwhelming. This enhanced the feasibility of long-distance communication such as conferencing and video telephony. A distinct element that facilitated the advanced communication competence of these systems is the presentation and estimation of emotions using the facial colors and expressions. Truly, emotion alters with the change of facial colors. These changes can be used for the fusion of the facial expressions to modify the basic emotions of visual anthrop-orphic agents. This may likely to 
play a pivotal role in selecting the communication between computers and humans $[1,2]$. The enhancement of the avatar's emotional expression by haptic sensation is reported [3]. Appearance of human facial colors is applied to influence the display of virtual facial image, emotional evaluation, individual identification and remote health care. This is regarded as one of the most peculiar manifestations among all human expressions[4]. Blushing is a common topic of psychological study and evidenced to be an important facial signal which serves vital functions in interpersonal communication [5]. Among psychologists, the explanations on human blushing still remain far from being understood. Most people consider blushing in public as an uncontrollable response and get embarrassment while they blush in public. Furthermore, blushing is a symptom that can make someone even worse suffering from social phobia [6,7]. Meanwhile, pallor effects due to shock, fear or pain occur through temporary cerebral anemia and contractions of the treatment capillaries in the face due to an increase in cerebral blood flow. This action diminishes the blood flow in the face and forwards it to the brain for relief and recovery. Despite much research, the accurate measure of pallor is not found yet. Psychophysiological analysis of pallor is expected to provide known results because the red areas are more likely of pallor and their time pattern. Recently, a formal monitoring reveals that the pallor received far less attention in the psychophysiological analysis compared with redness. The vital component of the computer model for the identification of the main characters centered towards this phenomenon. In our opinion, for vascular emotions the skin color or tone determines their occurrence [8]. The previous studies focused mainly on the geometric features of these alterations. For instance, the facial surface animation including skin stretching and wrinkle structures are widely investigated. Changes in hemoglobin concentration can cause alterations in skin colors. It can also originate from the reaction of histamine or other skin conditions such as blushing and rashes. Specifically, blushing consists of a number of emotions such as joy, shame and arousal. Regardless of their ability to convey emotion, the dynamic changes that occur in skin pigmentation are mostly ignored in the existing skin appearance models [9-11]. Reasonable behavior and realistic appearance is essential for simulating communicative behavior.

\section{Facial Animation}

Facial animation is attributed to be the most complex yet the most effective communication tool in the field of creative animation of virtual avatar or social agents [12]. Achieving high quality animation poses many challenges connected to the elements of the face including facial muscles, facial bones and synchronization of the lips in speech formation. Accurate and efficient identification of these elements require remarkable efforts and sufficient time for skilled animators. However, the knowledge of facial color animation is essential because facial expressions contain most of the information from which we recognize people's emotions and mental states. According to Thalmann [13], creation of believable facial animation is very significant because the human face is fundamental art object in understanding emotion. Hjortsjo (1969) developed the mimic language (ML) for facial expression and described the facial expression from the perspective of facial features, gestures and postures [14]. In ML, facial expressions are result from the combination of static structural and dynamic elements of the face such as the variation in form and appearance. The static aspects of the expressions are composed of the bone structure and soft tissues of the face. Conversely, the dynamic aspects of the facial expression originate from a mixture of soft facial features and changes in shape. In relation to this, Smith (2006) integrated the aspect of dynamic elements of the emotion state with individual's mental state [15]. Therefore, it is common to discuss different techniques introduced for facial animation. 


\subsection{Facial Animation Techniques}

The classification of facial animation techniques into different categories is often tricky because no specific boundary between two methods do exist. However, recent techniques are adopted for the syntheses of several methods to achieve improved results. This is known to change and couple with its archaic informal name conventions [16]. Traditional facial animation methods comprise of blend or shape interpolations called morph target or moving vertices, which are curves or meshes of 3D face geometry. One disadvantage of these methods is that only one facial region is sampled at a particular time. Conversely, it is well known that facial regions are intimately correlated to each other. To achieve realistic effects, animators need to be very careful around the edges of different facial regions. Natural representation of the details and creases are extremely hard. Newer technologies are emerging to improve the results by providing better intuitive dimensions in creating facial animations [17-19]. Currently, several types of facial animation techniques are available including mixed and group based animation systems [20].

\subsubsection{Shape Interpolation or Blend Shape}

Blend shape which is also considered as shape interpolation animation has become the most used animation method and is commonly used in commercial animation software packages. Packages such as MAYA and 3D Studio max are embedded in blend shape. Blend shape is achieved by shaping distortion while fading it into another through marking corresponding points and vectors on the "before" and "after" shapes which are used in the morph. The major concept of these animators is to create several key poses of a subject to automatically interpolate the frames in-between via animation. Technically, blend shape animation is a point set interpolation where a linear function specifies smooth motion between two sets of key points. Generally, it is from models of the same topology. This type of animation process includes modeling of polygonal meshes of a human face to approximate expressions. Besides, it also takes account of visemes for synchronizing the position of lips with its speed [21] and then continues with the automatic blending of different sub meshes called morphs targets or blend shapes. Since the blend shape method generally uses a linear algorithm of topology of the face meshes, animators can control the weights applied on the vertices of polygonal models. Thus, the degree of blending is accurately controllable [22]. Almost all of the automatically generated frames in this method are resulted from the simple transformation of scale, movement, or rotation of rigid objects. Examples of rigid objects or parts in human body are arms and legs. However, no specific rigid parts of the human face are known. Accordingly, this poses a difficulty for shaping interpolation in facial animation due to intricate construction and curves of the face. Indeed, matching of each face model by adjusting the settings is very expensive. Overall, it is harder to work with a real human facial model than a fantasy 3D facial character. Arbitrarily exaggerated expressions can be created on avatar's faces which may appear believable to people. For instance, the character Gollum in the movie "The Lord of the Rings" is an exaggerated expression. The rigid interpolations generated by the blend shape approach often impede the true nature of the animation in achieving realistic human facial emotional expressions. Several shape interpolation based methods are developed to get a smooth animation of the human face. One widely-used basic approach is the creation of a model face in the rest position. Then, using 3D modeling techniques, the points of a copy of that face are edited to make other faces. Typically, with the same topology the rested face is copied into different phoneme and expression states [23]. A typical facial animation using linear interpolation method is shown in Figure 1 (A)-(C). 


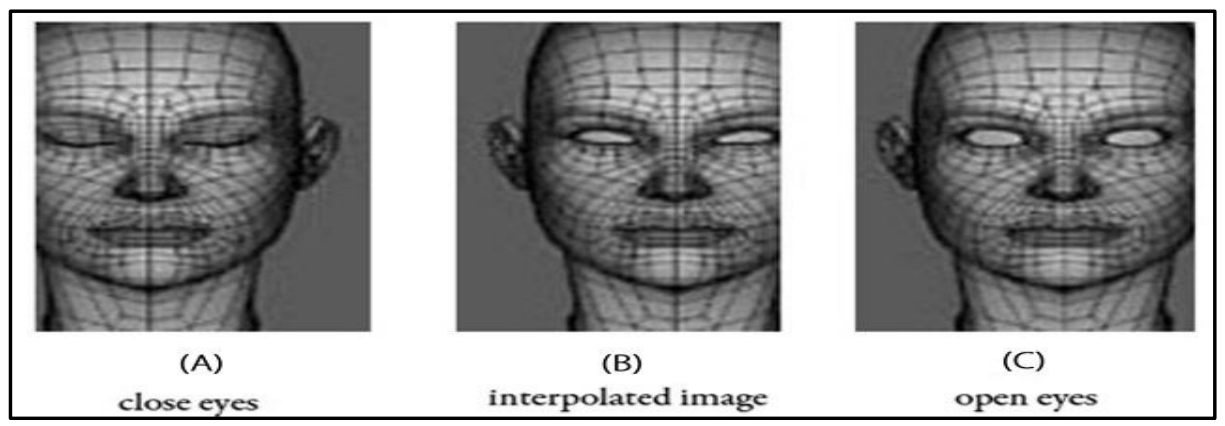

Figure 1. Linear Interpolation on Eyes from Close to Open with Interpolated _eyes $(t)=(1-t){ }^{*}$ closed_eyes $+t$ * open_eyes, where $t$ is between 0 and $1[22]$

\subsubsection{Parameterization}

The limitations and restrictions associated with simple interpolations can be overcome by parameterization because animators use parameter values to control expressions. The first facial animation system was designed by Fred Parke's from University of Utah and the NY Institute of Technology [24]. He used a control parameterized method where the animation turned into a process of specifying and controlling set of values of the parameter as a function of time. By a combination of independent parameter values, ideal parameterizations can identify any potential face and expression. The main limitation with shape interpolation method is that the interpolation process of the animation cannot be controlled by animators because the computer automatically manipulates it. Even though the computer calculates and provides a suitable interpolation, the animators' manual adjustments become limited. Through parameterization, animators can have more freedom and specific facial configurations control. By combining the parameters together, a wide range of facial expressions with quite low computational costs can be achieved. Yet, parameterization has one big restriction which limits its uses. Conflicts may occur because it usually works on small facial regions. For instance, there are obvious motion boundaries between the facial regions as the parameters can only affect certain areas. As a result, during parameter configurations, animators may not get enough freedom. Facelift program for "The Sims" uses a version of production system with hierarchical parameterization and genetic programming to improve the past procedures [25].

\subsubsection{Performance-driven Techniques}

Performance-driven approaches for human motion production in computer animation industry are subjective [26, 27]. Obviously, the biggest challenges in computer graphics of facial animation are related to the dynamic properties of face which are too many and the associated delicate emotions that cannot be performed by moving slide bars or dragging control points [28]. However, the best technique for the creation of facial animation has to emerge with the face itself meaning the capturing of movements directly from real faces and transferring the information digitally. This was first invented by Lance Williams using few markers on the face, a video camera to scan the face and track the markers [29]. Performance-based animation uses interactive input devices such as data gloves, instrumented body suits and laser or video-based motion tracking systems [24]. The system possesses a video camera that captures points on the performer's face which moves with the head. The camera receives the position of markers or dots drawn on the face and simultaneously tracks the movements of real performers in three-dimensional models. Presently, all tracking devices for face are based on optical or video technologies. System such as Vicon tracks above 100 markers with more precise and realistic facial animations. Although the capture process is unstable, it is consistent and accurately tracks the neces- 
sary processes. One achieves precise features of points and edges on the face by maintaining a great animation quality. The processed data from the camera can be used to create facial animation straight up or to infer AUs (Action Units) of the FACS technique. Performance-driven technologies are mainly classified in three categories including optical flow tracking, motion capture with or without markers and motion capture with key frame animation. The main challenges of these techniques are concerned with their data accuracies where the data from the systems need to be filtered before proceeding for animation. In this way animators can use the data-driven animations by setting errors. Motion capture is a very promising technique used for both commercial and academic purposes. Some of the most powerful facial animation techniques are Face Robot, Motion Builder, Crazy Talk, Zign Track, Facefx and Image Metrics. They own some striking features such as ease to handle, less time consuming and produce quite superior quality facial animations. The Motion capture techniques are in existence for the past few decades. For instance, the movie "The Polar Express" in 2004 implemented marker-less motion capture techniques to track the facial movements of all characters as illustrated in Figure 2 (A,B) Hundreds of motion points were captured for restructuring the realism [30-34].

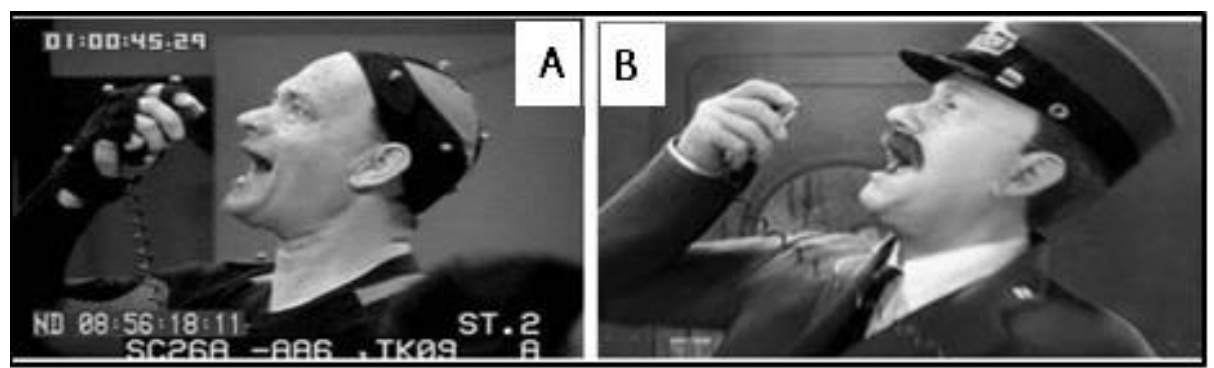

Figure 2. The Actor Tom Hanks (A) is Playing the Role of Conductor in the Film The Polar Express. (B) The Avatar is Driven by the Performance of the Actor[22]

\subsubsection{Transferring Existing Animations to Different Models}

The transfer of existing facial animations from a source model to a new target is the main concern. Different approaches are developed on model (cloning) due to tedious and time-consuming nature of the animation process. The easy replication of animations created by any tools or methods to new models allows animators to freely select from a large, great quality, facial and motions database animations. The animations can be reused and composed to new models even when there are differences. There is a tuning step for the new model after every transferred is made on a model specific animation which saves enormous labor costs. Performance-driven and data-driven animation can theoretically be regarded as specific ways of transferring. For this case the source used is the 2-D video footage. Furthermore, 2-D videos, existing 3-D animated face meshes and 3-D captured facial motion data can all be considered as the source even if the initial target is a 3-D face model [35]. Noh and Neumann in 2001 proposed an expression on cloning technique to create facial animations[36]. In their method, the transfer of existing facial animations to a new model is performed using the motion vectors of the vertex from the source model. This approach constructs a map from each vertex motion vector to a target model vertex even though the model contains different mesh geometry and vertex configuration. The mapping idea relies on the radial basis functions. Currently, many approaches are developed to construct the connections between the source and the target model for facile utilization of expression on cloning [37]. Pyun introduced a blend-shaped model containing 20 feature points and an algorithm to determine the displacement vector for the blending and tuning processes [38]. This model automatically extracts the feature points from the source for blending the facial expressions to the target model. However, proper blend- 
shape approaches require performing the action. They are not applicable on a predesigned blend-shape model because of the deficiencies of right configuration and setting.

\subsubsection{Eye Emotion Synthesis}

Facial animation must not only concern with the muscles of the face but must care the eyes which are crucial and subtle. Eye emotion is the powerful sign of the mental state and information manifestation of humans. People during talking sometime stare into each other eyes to sense the response in term of emotions, interest, information and attentiveness. Eyes being the "windows to the soul" gaze strong and delicate signals and make them significant for animation $[39,40]$. However, the creation of truthful talking animators is exceedingly complex. Audiences do not pay much attention to the lip synchronization once there is eyes communication. Truly, the entire animation and game industry is obsessed with the lip synchronization rather than eye motion. In animated character the eye animation is either non-existent or precisely ill-timed. The main reason for the lacking of eye animation may be attributed to the large eye movement. Eye gaze that arise partly due to the eyelid motions and the deformations do not reveal any deterministic correlation. Consequently, the direct motion capture data is unable to produce convincing and useful eye animation, instead fabricate the slight flicks inside the eyes. Methods with motion capture must be developed for achievable tracking of pupil and iris movement. Besides, the key frame for eye animation is too expensive. The captured data cannot directly be used prior to the key frame movements due to technical hurdle and performance related issues [41].

\subsubsection{Head Motion Synthesis}

Naturally, head motions play a vital role in making attractive interactions in humancomputer interface. Facial gestures possess significant and distinctive non-verbal information in human communication. For example, a basic nodding and rotation action of a 2D smiley face is often used to represent right or wrong in an online question game. This is easily understood by the user without any change on the smiley face. Therefore, head should serve as a model for producing realistic facial animations besides the lips, eyes and muscles for effectively mimicking facial behavior. Head movement is straight forward either nodding or rotating. The entire facial expression is taken into account by animators for creating head movements. Researchers developing such techniques must be aware of the existence of perfect correlation between head movement and the facial expression, and the usefulness of head movement to the audience in grasping the avatars mental state. However, a facial gesture is primarily attributed to face muscles movements, eye animation and long head movements. The combination of other factors in creating head motions is also considered [89]. Busso asserted that the head motion patterns (in movement activation range and velocity) with the neutral speech are significantly different from that with emotional speech. The presented HMM (Head Motion Synthesis) framework provided the best description of the dynamics of head motions and generated natural head motions directly from acoustic prosodic features. Figure 3 displays the overview of HMM-based head motion synthesis framework [42]. A mechanism for controlling head movement of photo realistic lifelike agents is developed by Hiroshi. The agents can exist in various modes such as idling, listening, speaking and singing [43]. The situation imitates the manner in which the head motion of a person reveals the agents virtual personalities. Graf analyzed quantitative head and facial movement accompanied by speech to demonstrate the relation to the text's prosodic structure [44]. The head movements are found vary depending on various verbal conditions. It is claimed that head movements strongly depend on different pitch accents and spoken text. Deng proposed a data-driven audio based head motion synthesis method to capture a real human face speaking. Variousaudio features were extracted and further analyzed using K-Nearest Neighbor (KNN) algorithm to syn- 
thesize the head motions using audio input data [45]. Next, we continue the discussion on the theory and colors of emotions.

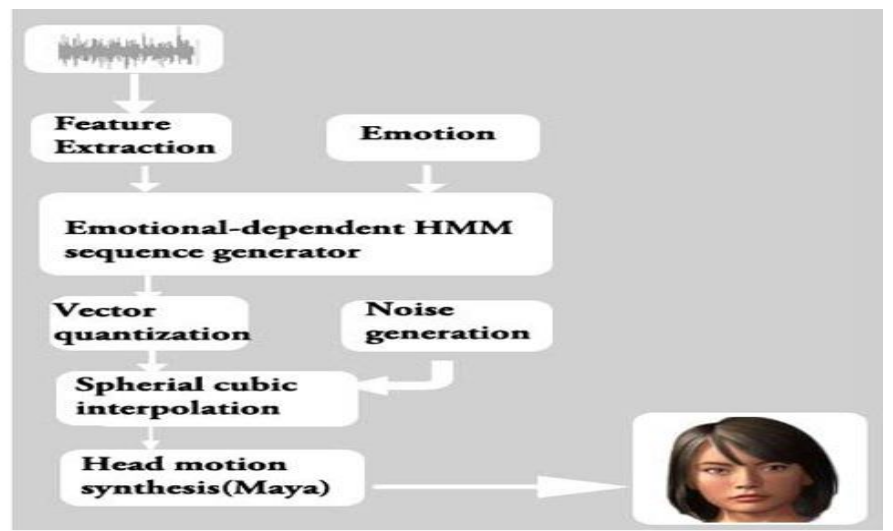

Figure 3. Schematic Diagram for HMM-based Head Motion Synthesis [42]

\section{Emotion Theory}

Despite outstanding research the relationships on the constituent of emotion and associated representation remain debatable. Kleinginna et al., [46] had compiled the definitions of emotions into more than 90 descriptions. Emotions are also described as the conditions of feelings $[47,48]$. A condition of feelings that may have positive or negative affective valence is described as automatic arousal [49]. Varieties of the definitions of emotion by Frijda[50]result an unclear score for terms such as "emotion", "feeling", "mood" and "attitude". Regardless of all these definitions the relationship between emotion and emotional stimuli remain ill-defined. Lopatovska and Arapakis [50] acknowledged that there are two categories of emotion theories. The first one involves cognition as an element that is necessary for emotion which can explain the expression of emotional events [51, 52]. This theory believes that cognitive activities may occur consciously or unconsciously, intentionally or unintentionally and in physical or intellectual aspect. Folkman et al., referred the activity as cognitive appraisal [53]. Besides, the activity is also linked to the assessment while encountering the surroundings and is important for individual's health. Lazarus mentioned that cognitive evaluation is important in the process of studying the meanings of stimuli and one can handle it. Meanwhile, Plutchik [54]introduced a new concept by combining emotion and animals' instincts. Ekman [55] endorsed this view and added that emotions transforms as time passes and this is caused by adoption values. He mentioned that the basic role of emotion is to mobilize organisms to quickly respond to those events which are identical to the past one [56]. In the theory of information studies both notions are used either directly or indirectly. Based on the somatic emotional theory, some studies are aimed to identify the participants' feelings on the assessment component from the emotional reaction [57-59].

\section{Emotion Color}

Emotions are complex states of mind. They include physiological correlation, cognitive factors and social roles [60]. Emotions function provides an individual with energy in order to react to a behavior. Different types of emotions exist and the basic one cannot be breakdown. Basic emotions which are regarded as universal can occur even though there are different personal experiences and cultural variations. They can be identified solely through facial expression. The six types of basic emotions are happiness, surprise, disgust, anger, sadness and fear. Even though facial expressions are unintentionally expressed but can be controlled through practice [61]. Micheal [62] stated that the facial expressions 
reflect social signal and manifest one's emotional states. Emotional states are manifested through facial expression and are commonly accompanied with color changes. These changes in color occur either with increasing or decreasing blood flow result rosiness or pallor. Colors play pivotal role for providing the viewer the information about the nature of objects. Any changes that occur to the objects are recorded by the observer. If the changes pertain to facial color then it must be linked to emotion. The correlation between emotion and color to human biology is verified. Parts of the colors can be redness (rosiness) or whiteness (pallor). There are varieties of colors to express emotion [61].

\section{5 .Theory of Emotion Colors}

This section includes the history and color theory from Goethe, descriptions of color meanings as suggested by Claudia Cortes and Naz Kaya. In addition, the opinions from Wheel pro and Shirley Willett regarding emotion colors in designing websites are provided al. A process example from Yan Xue is underscored.

\subsection{Color Theory of Johann Wolfgang von Goethe}

"Color Theory", a highly celebrated work by Johann Wolfgang von Goethe (2005) exhibited many remarkable points related to color meanings, especially in the "Sinnlichsittliche Wirkung der Farbe"[63]. He defined colors as minus/negative part and plus/positive part, where the plus part such as yellow, red-yellow (orange) and yellow-red (vermeil)are related to arousal, lively and ambitious. Conversely, the minus colors represented by blue, blue-red and red-blue signify restless, yielding and yearning. Table 1 illustrates the significance of the colors according to Goethe.

Table 1. Color Summary of Johann Wolfgang Von Goethe

\begin{tabular}{|c|c|c|c|}
\hline Emotion & Negative trait & Positive trait & Color \\
\hline Calm & & Neutral and Calm & Green \\
\hline Red blue & More restless & More active & Blue-red \\
\hline Faith & & Charm seriousness, & Red \\
\hline Joy & $\begin{array}{c}\text { Reined, Green and Un- } \\
\text { pleasant }\end{array}$ & Purity and Pleasant & Yellow \\
\hline Powerful & Irritating & Energetic & Yellow-red \\
\hline
\end{tabular}

\subsection{Color Model of Claudia Cortes}

Claudia Cortes established the characteristics of positive and negative colors attributes by summing up their associations with particular and described the relations of the colors. Even though it was difficult to extract colors meanings but an influence on the emotion implications and their grid locations are provided, which constituted the main elements of the model. The summary of those colors traits are listed in Table 2, where the overlap of traits is shown[63].

Table 2. Color Extraction by Claudia Cortes

\begin{tabular}{|c|c|c|c|}
\hline Emotion & Negative trait & Positive trait & Color \\
\hline Melancholic, introspec- & Sorrow, arrogant & Passive, leadership & Purple \\
\hline Greed, faith & Sick, greedy & Neutral, calm & Green \\
\hline Sadness, confident & Depressed & Traditional, faithful & Blue \\
\hline Anger, love & Embarrassed, offen- & Emotional, active & Red \\
\hline Happiness/joy, fear & Cautious & Energetic, lively & Yellow \\
\hline
\end{tabular}




\subsection{Color Model of Naz Kaya}

The difficulty in defining colors meanings and their interpretation can be found in the work of Naz Kayas [64]. The emotion and color combination with the peak of overall score from frequency test are summarized in Table 3 [65].

Table 3. Color Summary by Naz Kaya

\begin{tabular}{|c|c|}
\hline Emotion & Color with Munsell notation \\
\hline Annoyed, disgust & Green-yellow (2.5gy 8/10) \\
\hline Tired & Purple (5p 5/10) \\
\hline No emotion, excited, energetic & Yellow-red (5yr 7/12) \\
\hline Loved, anger & Red (5r 5/14) \\
\hline Peaceful, hopeful, comfortable & Green $(2.5 \mathrm{~g} \mathrm{5/10)}$ \\
\hline
\end{tabular}

\subsection{Colors from Wheel Pro}

The commercial program Color Wheel Pro that has contributed to the definitions of colors meanings and associated traits is furnished in Table 4 [65]. In fact, there are overlapping traits commonly depending on their context and their exact meanings are dubious. The most appropriate words based on the meanings of the colors are tabulated.

Table 4. Definitions and Attributes from Color Wheel Pro

\begin{tabular}{|c|c|c|c|}
\hline Emotion & Negative trait & Positive trait & Color \\
\hline Power & Death & Elegance & Black \\
\hline Sadness & Frustration & $\begin{array}{c}\text { Romantic, nostal- } \\
\text { gic }\end{array}$ & Purple \\
\hline (Not given) & Power & Safety, purity & White \\
\hline Aggressive & Courage & Passion & Red \\
\hline Joy, happiness & Distrust,domination & Desire, wisdom & Orange \\
\hline
\end{tabular}

\subsection{Model of Shirley Willett}

Table 5 represents the colors and their characteristics from the perspective of Shirley Willett. The colors and their properties act as the standard for the relationship between basic emotions and colors. [64].

Table 5. Colors and the Associated Traits by Shirley Willett

\begin{tabular}{|c|c|c|c|}
\hline Emotion & Negative trait & Positive trait & Color \\
\hline Greed & Hoarding & Satisfaction & Green \\
\hline Confusion & Racing & Clarity & Blue \\
\hline Anger & Rage & Enthusiasm & Red \\
\hline Fear & Panic & Awareness & Yellow \\
\hline Shame & Disgrace & Pride & Orange \\
\hline
\end{tabular}

\subsection{Theory of Yan Xue}

Yan Xue had developed a model based on the colors and used Russell circumflex model as a basis as depicted in Figure 4[65]. He demonstrated the utility of color support in 
his work on Philips ICat. The ICat used blue, red and green to produce LED colors for placing them in feet and ears.

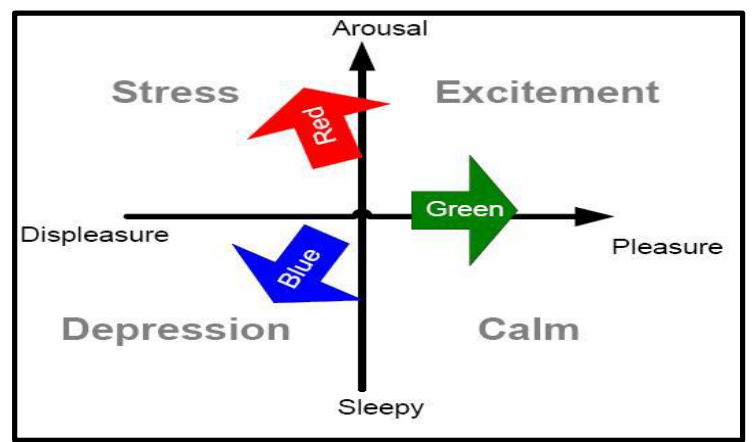

Figure 4. The Color Distribution used by Yan Xue[65]

\subsection{Comparison of Different Emotion Colors Models}

This section compares the merits and demerits of different emotion color models in detail discussion. The interpretation of Naz Kaya has tremendously contributed in delivering the colors meanings and their implications. The model of Claudia Cortes supports these interpretations for the basic colors. The complexity of Goethe's theory makes it difficult for implementation because the truthful of the information is tough to verify. Nevertheless, the model of Shirley Willet is highly useful in providing meanings of emotion colors. Finally, Yan Xue model renders a simplistic interpretation of three basic colors RGB. Interestingly, the basic emotion "surprise" does not have any color. The following discussion will be synthesis, analyses and appearance of facial colors.

\section{6 .Facial Color Appearance}

Facial color appearance models became remarkably popular in sports and film industry. Facial appearance (expression) changes regularly during speeches, exercising and emotional moments. Reproduction of facial expression in real time is quiet challenging because people are not only conscious but very sensitive to the look of their skin. Most facial color appearance models of skin possess two layered translucent structure and relates the expression to the distribution of melanin and hemoglobin in the blood which is well confirmed [66-68].

\subsection{Image Based on Skin Color Analysis and Synthesis}

Capturing the skin image for reproducing the colors depends on analysis and synthesis. This provides highly realistic representations for human faces because effects such as self-shadowing, inter-reflections and subsurface scattering are implicitly captured. Pighin et al. reproduced faces using view-dependent texture mapping [69] under static illumination conditions. Recent efforts on facial imaging allowed variations in lighting for static faces [70, 71], expressions[72] and real-time performances[73]. Debevec et al., introduced relight able 3-D face models by mapping image-based reflectance characteristics onto 3-D scanned geometry [71]. The use color space analysis is implemented to separate the image data into specular and diffuse components to extrapolate into new view. While their method looks into the aggregate behavior of subsurface scattering, specific diffusion parameter is not inbuilt. Therefore, this model is incapable of producing correct subsurface scattering effects neither for close up light sources nor for high-frequency spatially varying illumination. Borshukov and Lewis achieved subsurface scattering by combining an analytic surface BRDF (Bidirectional Reflectance Distribution Function) with an image-space approximation and created great real face models for the movie industry. Sand- 
er et al., developed a variant of this method for real-time face rendering on modern graphics hardware. Tsumura et al., illustrated a single skin image of hemoglobin extraction and melanin pigmentations shown in Figure 5 (A-C).

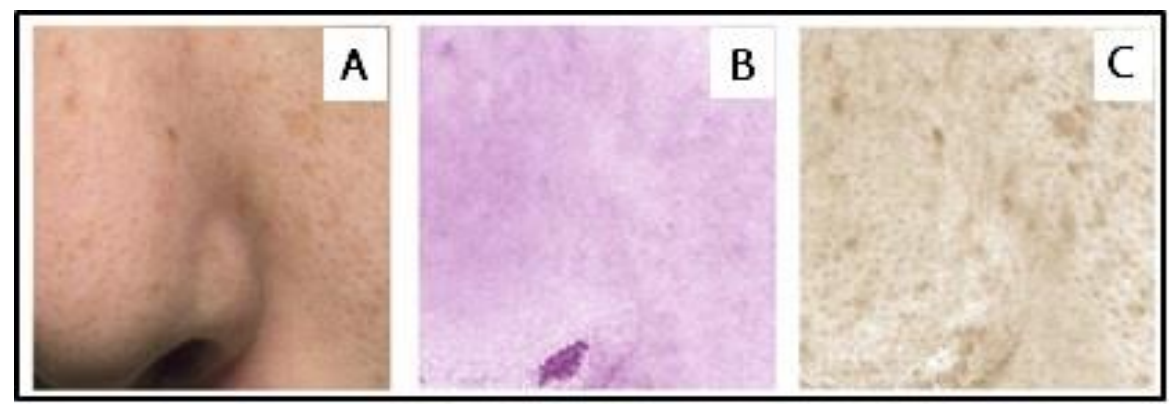

\section{Figure 5. Melanin and Hemoglobin Maps Extracted from a Single Image us- ing Independent Component Analysis(A): Original Image, (B): Melanin and(C): Hemoglobin)[74]}

An image-based model requires huge data storage systems, hard to edit and the dimensions are not highly physical in terms of light-skin interaction. Simulation of local light sources and reproduction of spatially-varying illumination remain a difficult task[75] especially when the shadows from distant sources do not agree with the shadows from local one. For instance, when a light source close to the nose is illuminated then an imagebased method would foresee zero reflection as the light from distant source is blocked by the nose. The introduction of reflectance based models provides further thrust towards the development of skin colors appearances.

\subsection{Reflectance Based Model}

The skin color appearance is known to differ depending on the lighting, viewing conditions and the direction of reflection on the skin. Varieties of skin reflection models from simple BRDF approximations to the light scattering $[76,77]$ and methods that simulate small-scale anatomy and physiology details [78] exist. The former one is based on the approximation of 1-D light transport which does not capture the characteristic subsurface scattering of the translucent materials. Models relying on an accurate volumetric light transport simulation are naturally too complex and very slow for real-life graphics applications. Furthermore, models based on the diffusion approximation offer a compromise between accuracy and complexity. Donner, et al. introduced a simple approach using the diffusion dipole approximation to evaluate optical parameters of materials including two samples of the skin [79]. This method is limited to homogeneous and semi-infinite slabs with regular scattering and absorption properties. Despite the limitation of the dipole model in approximating the appearance of heterogeneous materials it still remain active. Jensen and Buhler used the translucent model and varied other parameters to match a given albedo texture [80]. Later on, the method is also adopted by Hery [81]. Several workers successfully exploited the dipole model with fully spatially varying parameters [82]. However, the use of a model in the presence of heterogeneity is not properly defined particularly when large variations in parameters occur within small area. Most of the models considered the transportation of light between two points on the material because it depends only on a single set of homogeneous parameters. Weyrich et al., analyzed the variations in the reflectance of facial skin under 149 topics subjected to various external conditions. The parameters of skin reflectance are calculated by spatially varying subsurface scattering, texture and specular reflection [83]. Donner and Jensen introduced the diffusion multi-pole approximation to interpret thin slabs and multi-layered translucent materials [66]. Their model was able to capture the accurate reflectance of translucent layered 
materials but could not obtain optical parameters for precise materials. Subsequently, the multi-pole approximation was purposely employed in the creation of spectral shading model for the skin where a minor set of chromophore parameters were used to regulate the overall skin appearance. D'Eon et al., (2007) employed the multipole model to run contemporary graphics hardware [84]. Spatial variations on the surface are again approximated with an albedo modulated texture to interpret interactive layered translucent materials. General heterogeneous bidirectional surface scattering reflectance distribution function (BSSRDF) models are developed with the precise target of obtaining parameters from measurements [85-87]. These models identified the relationship between incident and extant light but left out the physical key and physiological structure of the material. For the skin, it is important to understand the relationship between the structure, pigmentation and reflectance to derive the physical models for forecasting the appearance of arbitrary skin samples. Wang et al., demonstrated a volumetric representation on heterogeneously scattered materials via a mathematical method [88]. The heterogeneous diffusion on the GPU is considered even though it was difficult to discretize the geometry to coupled poly-grid. Donner et al., simulated the skin reflectance by considering the light interscattering between skin layers[67]. The applied method called chromophore spectra derived the spatial chromophore distributions from multi-spectral photographs of skin. Ghosh et al., [89] considered both structured light and polarization to obtain the skin layer properties using a multi-layered scattering model.

\subsection{Bidirectional Texture Function}

Determining the skin appearance at finer scale is often complicated as it contains several color variations from pores, wrinkles, spots and freckles. A comprehensive model of skin appearance must therefore consider all the skin textures. The bidirectional texture function (BTF)introduced by Cula et al., [90] is a very useful concept for modeling the appearance of texture. The considered the variations in the appearance are related to the changes in lighting and viewing direction as shown in Figure 6. Cula et al., applied captured images of the skin while varying the viewing and lighting locations. The images obtained from different body regions differ because the skin texture varies according to body region, age and gender. A distinctive Skin Texture Database (STD) with high resolution images of several skin types is established [91].

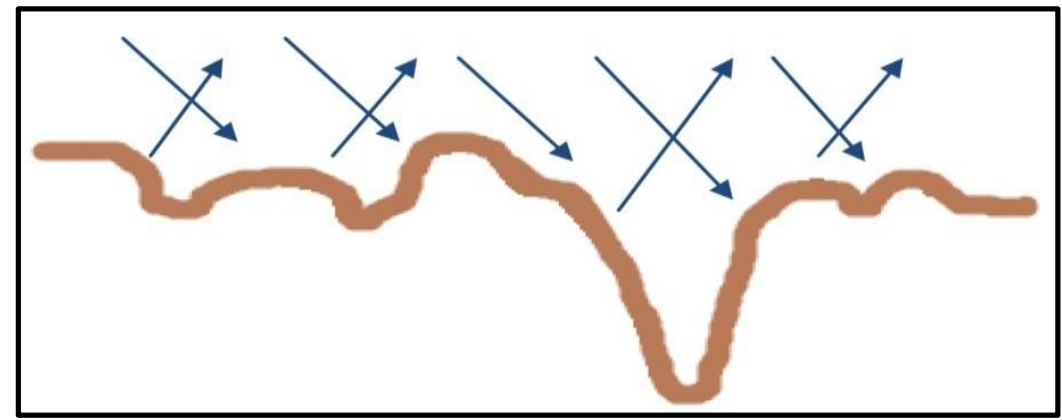

Figure 6. Illustration of Bidirectional Texture Function[91]

\subsection{Physiology Measurement Based Models}

In dermatology, physiological measurement methods are applied to blood hemoglobin samples for ex vivo histological examination or in vivo non-invasive point measures [92] as shown in Figure 7(A-C). 


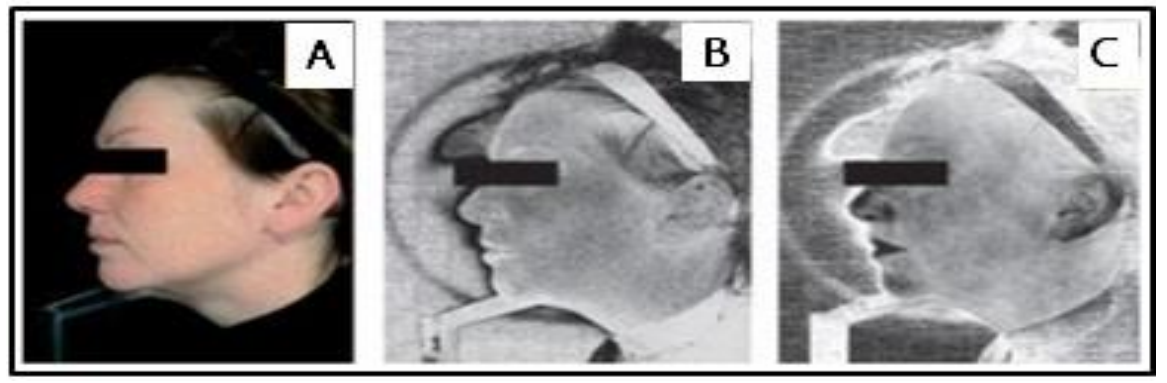

Figure 7. Example of Noncontact Point Measure Chromophore Map. (A): Original Digital Photo, (B): Melanin Concentration Map and (C): Hemoglobin Concentration Map [92]

Lately, Jimenez et al., demonstrated similar non-invasive procedures for in-vivo mapping of hemoglobin concentration and distribution across wide areas of the skin. They relate the change of hemoglobin with dynamic facial expression [10].

\subsection{Emotional Appearance Models}

Presently, the simulation of emotional appearance of the skin for different facial expressions is routinely performed [93]. The simulation models are user friendly even though they do not correlate real to actual hemoglobin or melanin maps. Kalra and Thalmann simulated changes in the skin for redness and pallor[8]. Yamada and Watanabe examined blood flux based on anger and dislike[94]. They measured concurrent changes in facial skin temperature and color for these emotions and mapped them into an average image for facial color expressions. Jung et al. demonstrated the skin colors changes based on complex parameter sets using fourteen states of emotions[95]. Melo and Gratch simulated blushing directly on a user-defined color change to different areas of the face [96].

\section{7 .Facial Expressions}

In Psychology, the in-depth analyses of facial expression generated renewed interests for decades. The attraction in this area is centered towards the understanding of human's information transmission and receives using their faces. It also intends to unfold the effects of mental and physical disabilities on facial movements. The expression on the faces of patients suffering from depression is often undergoes through a number of stages relating to their illness. It is hoped that breakthroughs in the treatment of patients may occur via facial monitoring of illnesses. Another area of interest to psychologists is the facial deception, which identifies whether a person is lying or not. When somebody is lying, it is easy to notice some conflict in the appearance on the face because there is more than one visible expression gets manifested on the face. Perception comes naturally and by studying the agreements and differences in movements with each other it is possible to interpret facial expressions [97-99].

\subsection{Facial Action Coding System}

Facial action coding system (FACS) is a scheme developed by Paul Ekman and Wallace Friesen that measures and describes the facial behaviors by understanding the texture, movement and anatomy of every facial muscle [12]. FACS is a known standard that demonstrates how each facial muscle is responsible for changing the facial appearance. It is derived from a facial anatomy analysis, which describes human face's muscles conduct including the movements of the tongue and jaw. Through exploring facial anatomy, one concludes that the changes in facial expressions are caused by facial actions. FACS starts working from facial actions to understand facial behaviors. Facial action units (AUs) are 
made in accordance to these actions and every AU involves numerous facial muscles. When an expression is made, the involved muscles cannot be recognized easily. Conversely, each muscle is divided by two or more AUs to explain quite self-governing actions of various muscle parts. FACS divides the human face into 46 action units. Every unit embodies an individual muscle action or a group of muscles that characterize a single facial position. The principle is that every AU is the smallest unit which cannot be reduced into minor action units. Nevertheless, by accurately sorting out various AUs on the face, FACS is capable of mimicking all the facial muscle movements[100]. The creation of facial appearance is the combination of action units that produce altered facial expressions. For example, combination of AU4 (Brow Raiser), AU15 (Lip Corner Depressor), AU1 (Inner Brow Raiser) and AU23 (Lip Tightened) generates a sad expression. Originally, FACS was not introduced for generating facial animations but it was rather aimed in describing the score in facial movements. Recently, the widespread usage and overwhelming acknowledgement for facial actions enables it useful exploitation in facial animation. FACS assists animators to exemplify and build realistic facial expressions by configuring all possible facial animation units of human face with the descriptions of measuring the positions of head and eye [22]. Unlike other muscle-based techniques, FACS studies the actions that are caused by the muscles instead of investigating the muscle directly[101]. The facial expressions are complicated because no expression is made by only one muscle. In fact, a single muscle is responsible for the creation of various expressions. The overall configuration and mechanism is very complex. Moreover, study of each muscle individually to facilitate a clear comprehension in facial action and subsequent expressions would be extremely difficult. FACS is very effective in distinguishing the actions of upper facial parts, but not the entire face. Particularly, FACS does not count for all of the visible, distinguishable and reliable actions of the muscle controls in the lower facial parts which make it unable to recognize the lip-synchronization requirements. Generally, FACS describes most expressions and movements by facial action coding. It offers very dependable face codes on the upper areas of the face including foreheads, eyelids and eyebrows. Nonetheless, FACS overcomes the limitation of interpolation and is a landmark for facial animation methods. It is extensively used with muscle or simulated muscle-based approaches. The explanations for signal sampling AUs are furnished in Table 6 and Table 7 displays the generation of the basic expression based on facial action.

Table 6. Representation for the Signal Sample Action Units

\begin{tabular}{|l|l|l|l|l|l|}
\hline AU & FACS Name & AU & FACS Name & AU & FACS Name \\
\hline 6 & $\begin{array}{l}\text { Check Rais- } \\
\text { er }\end{array}$ & 23 & $\begin{array}{l}\text { Lip Tight- } \\
\text { ened }\end{array}$ & 15 & $\begin{array}{l}\text { Lip Corner De- } \\
\text { pressor }\end{array}$ \\
\hline 1 & $\begin{array}{l}\text { Inner Brow } \\
\text { Raiser }\end{array}$ & 26 & Jaw Drop & 5 & Upper Lid Raiser \\
\hline 17 & Raiser Chin & 20 & Lip Stretcher & 9 & Nose Wrinkle \\
\hline 4 & Brow Lower & 14 & Dimpled & 16 & Lower Lip Depressor \\
\hline 10 & $\begin{array}{l}\text { Upper Lip } \\
\text { Raiser }\end{array}$ & 2 & $\begin{array}{l}\text { Outer Brow } \\
\text { Raiser }\end{array}$ & 12 & Lid Corner Puller \\
\hline
\end{tabular}




\section{Table 7. Our Explanation for the Generation of the Basic Expression based} on FACS

\begin{tabular}{|l|l|}
\hline Expressions & Involved AUs \\
\hline Anger & AU $(26+4+17+10+9+20+2)$ \\
\hline Fear & AU $(2+4+5+26+15+20+1)$ \\
\hline Happiness & AU $(14+12+6+1)$ \\
\hline Sad & AU $(23+1+15+4)$ \\
\hline Surprise & AU $(1+2+5+15+16+20+26)$ \\
\hline
\end{tabular}

\subsection{Moving Picture Experts Group}

The moving picture experts group (MPEG-4) is an ISO standard for multimedia MPEG41997. Since its release in 1999, many researches are concentrated on this standard because it can be used in a wide range of video and audio recording as well as in 3D graphics. The MPEG-4 being the only reliable standard dealing with facial animation is used as a basis for the development of new methods. Several investigations in the area of facial animation are carried out to describe and represent precise facial actions with predefined groups of "codes".

The FACS is perhaps the first prosperous effort in this regard even though not directly related to animation and graphics research. Lately, the MPEG-4 standard is focusing more on the integration and communication aspects of multimedia contents and is getting further attention in facial animation areas [102]. MPEG-4 Facial Animation (FA) outlines many parameters of a talking face in a standardized technique. It successfully describes the Face Definition and Animation Parameters for facial action encoding. In MPEG-4, the head is grouped into 84 feature points (FPs) in which every such point describes the shape of an area. They are used to outline the parameters of animation on the face to conform the standard when switching between other models. For the current standard, there are 68 universal Facial Animation Parameters (FAP). Every FAP corresponds to a FP and expresses the low level facial actions like jaw-down and more complex higher level actions such as smiling. The FAP being independent of model geometry requires systematic calibration before it is applied on different models. This is achieved by using FAP units (FAPU) which is defined as a distance between facial features and is not universal. It is exclusive to the 3D face model where ever applied. Therefore, when a standard FAP with corresponding FPs and FAPU are available the values can be adjusted and decided on a new model freely by exchanging information from the face models. They together permit a receiver system to produce a facial animation by using any graphics method based on low level commands in FAPs. The FAPs consist of two categories in which the first one represents the facial expressions by themselves with high level considerations. It includes six basic emotions such as anger, sadness, surprise, joy, fear and disgust. Two parameters are only allowed to be used by animators to create a relatively good animation by performing linear interpolation between each expression. The other parameters of facial animation focus on certain facial areas, for example, the left corner of mouth, bottom of the chin and the right eyebrow, to cite a few. This can encode a higher degree of facial actions with more sophistication. It is important to mention that FAPs do not require any geometric models and synthetic face to be used. They simply sketch the desired movements and are independent of methods of animation. They can be utilized in applying pre-learned image transformations on the basis of detecting locations of facial features to a real 2D picture. This in turn generates visual effects like facial expression, talking, or any further facial movements. Furthermore, MPEG-4 delivers a scripting language capable of producing parameters[103, 104]. 


\section{Conclusion}

We provide a detailed overview on facial animation techniques signifying theories and models for emotional expression and facial colors. The past development, present art of research in implementation and future prospects of facial skin colors on 3D avatar is underlined. Theory of emotion that includes emotion color as subtype with several models of emotional colors is emphasized. Manifestations of facial expressions are described using FACS and MPEG.

Several prominent facial animation techniques such as blend shape interpolation, parameterization, performance driven processes, transfer existing animations to different models, eye emotion synthesis and head motion synthesis with their overwhelming success and limitations are underscored. The urgent need of facial animation in the entertainment industry, telecommunication medicine and many others are stressed. It is recognized that the future advancements of facial animation would be mostly guided by computer graphic technologies. The new forms of motion capture techniques are developed as the dominant approaches for performing fast, flexible and realistic animations. The significance of colors in providing the information about the nature of objects, stimulation to the viewer to respond to a group of circumstances and their close relations to emotion are highlighted via the example of Felice Casson court proceedings. The color theory confirms the strong correlation between emotion and human biological responses. Varieties of colors exist to express emotion and a part is manifested as redness (blushing) or whiteness (pallor). Defining the precise correspondence rules between emotions and colors will remain a challenge. The current facial expression of virtual human is still lacking in terms of providing realistic appearance through their facial skin colors and texture changes. This will continue to encourage in performing better color classification to determine the facial skin colors changes of the avatar. The established methods are still limited in generating colors for synchronizing real skin color changes. Their RGB properties are still deficient on representing facial color changes since the skin colors are mostly affected by oxygenation and de-oxygenation of hemoglobin. The mechanism of oxygenation and de-oxygenation in determining the facial skin colors changes will remain future key issue. This exhaustive and informative review article is believed can serve as taxonomy for a better understanding the research advancements towards human facial skin colors on 3D avatar.

\section{Acknowledgements}

Authors are grateful to Universiti teknologi Malaysia, Research Management Centre (RMC) of UTM, Department of Informatics, Faculty of Information Technology, Institut Teknologi Sepuluh Nopember Surabaya, Indonesia.

\section{References}

[1] T. Yamada and T. Watanabe, "Effects of Facial Color on Virtual Facial Image Synthesis for Dynamic Facial Color and Expression Under Laughing Emotion", The 13th IEEE International Workshop of Robot and Human Interactive Communication, ROMAN, (2004) September 20-22; Kurashiki, Okayama Japan.

[2] T. Wu, S. Fu, and G. Yang, "Survey of the Facial Expression Recognition Research, in Advances in Brain Inspired Cognitive Systems",Springer Berlin Heidelberg vol. 7366, (2012), pp. 392-402.

[3] A. H Basori, D. Daman, A. Bade, M. S. Sunar and N. Saari, "The Feasibility of Human Haptic Emotion as a Feature to Enhance Interactivity and Immersiveness", Virtual Reality Game Proceedings of the 7th ACM SIGGRAPH International Conference on Virtual-Reality Continuum and Its Applications in Industry, (2008) December 8-9; Singapore. 
[4] A. H. Basori, A. Bade, M. S. Sunar, N. Saari, D. Daman and M. D. H. Salam, "An Integration Framework for Haptic Feedback to Improve Facial Expression on VirtualHuman",International Journal of Innovative Computing, Information and Control. 8, 11 (2012).

[5] C. Darwin, Editor, "The Expression of the Emotions in Man and Animals", John Murray, London (1872).

[6] P. Ekman and W. V. Friesen, "Detecting Deception from the Body or Face", Journal of Personality and Social Psychology, vol. 29, no. 3 (1974). pp. 288-298

[7] J. W. Gooch, "Blushing, in Encyclopedic Dictionary of Polymers", Springer New York. (2007), pp. 8788.

[8] P. Kalra and N. Magnenat-Thalmann, "Modeling of Vascular Expressions in Facial Animation", Proceedings of Computer Animation '94, (1994) May 25-28.

[9] N. Ersotelos and F. Dong, "Building Highly Realistic Facial Modeling and Animation: A Survey", The Visual Computer, vol. 24, no. 1, (2008), pp. 13-30.

[10] J. Jimenez, T. Scully, N. Barbosa, C. Donner, X. Alvarez, T. Vieira, P. Matts, V. Orvalho, D. Gutierrez and T. Weyrich, "A Practical Appearance model for Dynamic Facial Color", Proceedings of ACM SIGGRAPH Asia,vol. 29, no. 6 (2010).

[11] S.-Y. Yoon and K. Wise, "Reading Emotion of Color Environments: Computer Simulations with SelfReports and Physiological Signals", Industrial Applications of Affective Engineering, Springer International Publishing, Switzerland (2012), pp. 219-232.

[12] R. J. S. Sloan, B. Robinson, K. Scott-Brown, F. Moore and M. Cook,"A Practice-led Approach to Facial Animation Research", Proceedings of the SSPNET 2nd International Symposium on Facial Analysis and Animation, (2010) October 21; Edinburgh, United Kingdom.

[13] N. Magnenat-Thalmann, H. Kim, A. Egges and S. Garchery, "Believability and Interaction in Virtual Worlds", International Multi-Media Modeling Conference, (2005) January 12-14; Melbourne, Australia.

[14] W. Musterle and O. E. Rossler, "Computer faces: The Human Lorenz Matrix", BioSystems, vol. 19, no. 1, (1986).

[15] A. P. Smith, "Muscle-Based Facial Animation using Blend shapes in Superposition", (2006).

[16] B. Bianchi, A. Ferri, S. Ferrari, C. Copelli, L. Salvagni and E. Sesenna, "The Masseteric Nerve: A Versatile Power Source in Facial Animation Techniques", British Journal of Oral and Maxillofacial Surgery, vol. 52, no. 3, (2014).

[17] H. Zhou and S.P. Mudur, "3D Scan-based Animation Techniques for Chinese Opera Facial Expression Documentation", Computers \& Graphics, vol. 31, no. 6, (2007), pp. 788-799.

[18] R. Parent, "Computer Animation: Algorithms and Techniques", Newnes, (2012).

[19] I. Kerlow, "The Art of 3D: Computer Animation and Effects", John Wiley \& Sons (2004).

[20] A. H. Basori and A.Z. Qasim, "Extreme Expression of Sweating in 3D Virtual Human", Computers in Human Behavior, vol. 35, (2014), pp. 307-314.

[21] J. Osipa, Stop Staring: Facial Modeling and Animation Done Right. John Wiley \& Sons, (2007).

[22] C. Liu. "An Analysis of the Current and Future State of 3D Facial Animation Techniques and Systems", B.A., Communication University of China: Canada, (2009).

[23] M. Abbas, A. A. Majid, and J. M. Ali, "Positivity-preserving Rational Bi-cubic Spline Interpolation for 3D Positive Data", Applied Mathematics and Computation, vol. 234 (2014), pp. 460-476.

[24] P K. Parke, K. Waters, and T. R. Alley, "Computer Facial Animation",AK Peters Wellesley, (2008).

[25] S. DiPaola, "Exploring a Parameterised Portrait Painting Space", International Journal of Arts and Technology,vol. 2, no. 1, (2009), pp. 82-93.

[26] F. I. Parke, K. Waters, and T. R. Alley, Computer Facial Animation. A. K. Peters, Ltd (1996).

[27] Y. Chen, G. Dang, Z.-Q. Cheng and K. Xu, "Fast Capture of Personalized Avatar Using Two Kinects", Journal of Manufacturing Systems, vol. 33, no. 1, (2014), pp. 233-240.

[28] J. Troya, A. Vallecillo, F. Durán and S. Zschaler, "Model-driven Performance Analysis of Rule-based Domain Specific Visual Models", Information and Software Technology, vol. 55, no. 1, (2013). pp. 88110 .

[29] L. Williams, "Performance-driven Facial Animation", Proceedings of the 17th ACM Conference of Computer Graphics \& Interactive Technique, (1990) August 6-10; Dallas, TX, USA.

[30] Y. Furukawa and J. Ponce, "Dense 3d Motion Capture for Human Faces", IEEE Conference of Computer Vision and Pattern Recognition, (2009) June 20-25; Miami, FL, USA.

[31] P. Kaimakis and J. Lasenby, "Markerless Motion Capture with Single and Multiple Cameras", IEEE International Conference of Image Processing, (2004) October 24-27; Singapore.

[32] B. Rosenhahn and T. Brox, "Scaled Motion Dynamics for Markerless Motion Capture", IEEE Conference of Computer Vision and Pattern Recognition, (2007) June 17-22; Minneapolis, Minnesota, USA.

[33] B. Rosenhahn, C. Schmaltz, T. Brox, J. Weickert, D. Cremers and H. -P. Seidel "Markerless Motion Capture of Man-machine Interaction", IEEE Conference of Computer Vision and Pattern Recognition, (2008) June 23-28; Anchorage, Alaska, USA.

[34] A. Sundaresan and R. Chellappa. Markerless Motion Capture Using Multiple Cameras, "Computer Vision for Interactive and Intelligent Environment", IEEE, (2005) Nov. 17-18; USA. 
[35] V. Karavirta, A. Korhonen, L. Malmi and T. Naps, "A Comprehensive Taxonomy of Algorithm Animation Languages", Journal of Visual Languages \& Computing, vol.21, no. 1 (2010). pp. 1-22.

[36] J.-Y. Noh and U. Neumann, "Expression Cloning", Proceedings of the 28th annual conference on Computer graphics and interactive techniques, ACM, (2001) August 12-17; Los Angeles, CA, USA.

[37] Y. Zhang, W. Line, B. Zhou, Z. Chen, B. Sheng and J. Wu, "Facial Expression Cloning With Elastic and Muscle Models", Journal of Visual Communication and Image Representation, vol. 25, no. 5 (2014), pp. 916-927.

[38] H. Pyun, Y. Kim, W. Chae, H. W. Kang and S. Y. Shin, "An Example-Based Approach for Facial Expression Cloning", Proceedings of the 2003 ACM SIGGRAPH/Eurographics symposium on Computer animation, Eurographics Association, (2003) July 26-27; San Diego, CA, USA.

[39] H. Kacorri, A. Harper, and M. Huenerfauth, "Comparing Native Signers' Perception of American Sign Language Animations and Videos via Eye Tracking", Proceedings of the 15th International ACM SIGACCESS Conference on Computers and Accessibility, ACM, (2013) October 21-23; Bellevue, WA, USA.

[40] B. Schuller, F. Eyben and M. Pantic, "The Continuous Audio/Visual Emotion Challenge", Proceedings of the 14th ACM international conference on Multimodal Interaction, ACM, (2012) October 22-26; Santa Monica, California.

[41] N. Perrett, Chaning the Face of Animation, (2008).

[42] C. Busso, Z. Deng, M. Grimm, U. Neumann and S. Narayanan, "Rigid Head Motion in Expressive Speech Animation: Analysis and Synthesis", IEEE Transaction Audio, Speech, and Language Processing, vol. 15, no. 3, (2007), pp. 1075-1086.

[43] J. P. Lewis, J. Mooser, Z. Deng and U. Neumann, "Reducing Blendshape Interference by Selected Motion Attenuation", Proceedings of the 2005 Symposium on Interactive 3D graphics and Games, ACM, (2005) April 3-6; Washington, DC, USA.

[44] H. P. Graf, E. Cosatto, V. Strom and F. J. Huang, "Visual Prosody: Facial Movements Accompanying Speech", Proceedings of Fifth IEEE International Conference Automatic Face and Gesture Recognition, (2002) May 20-21; Washington, DC, USA.

[45] Z. Deng, J. P. Lewis, and U. Neumann, "Automated Eye Motion Using Texture Synthesis", Computer Graphics and Applications, IEEE, vol. 25, no. 2, (2005), pp. 24-30.

[46] P. R. Kleinginna Jr. and A. M. Kleinginna, "A Categorized List of Motivation Definitions, With a Suggestion for a Consensual Definition", Motivation and Emotion, vol. 5, no. 3, (1981). pp. 263-291.

[47] P.N. Johnson-Laird and K. Oatley, "The Language of Emotions: An Analysis of a Semantic Field", Cognition \& Emotion, vol. 3, no. 2, (1989), pp. 81-123.

[48] A. Ortony, G. L. Clore, and A. Collins, "The Cognitive Structure of Emotions", Cambridge University Press (1990).

[49] S. Schachter and J. Singer, "Cognitive, Social and Physiological Determinants Of Emotional State", Psychological Review, vol. 69, no. 5 (1962), pp. 379-399.

[50] N. H. Frijda, "The Emotions", Cambridge University Press, (1986)

[51] K. Oatley and P. N. Johnson-Laird, "Cognitive Approaches to Emotions, "Trends in Cognitive Sciences", vol. 18, no. 2, (2014), pp. 134-140.

[52] A. Gangemi, F. Mancini, and P. N. Johnson-Laird, "Models and Cognitive Change in Psychopathology", Journal of Cognitive Psychology, vol. 25, no. 2, (2012), pp. 157-164.

[53] S. Folkman, R. S. Lazarus, R. J. Gruen and A. DeLongis, "Appraisal, Coping, Health Status, and Psychological Symptoms. Journal of Personality and Social Psychology", vol. 50, no. 3, (1986). pp. 571.

[54] R. Plutchik, "A General Psychoevolutionary in Theory of Emotion", Emotion: Theory, Research, and Experience, Edited R. Plutchik and H. Kelleman, (1980), vol. 1, no. 3, pp. 3-33.

[55] P. Ekman, "Expression And The Nature of Emotion", in Approaches to Emotion, Edited K. R. Scherer amd P. Ekman, Psychology Press (1984), vol. 3, pp. 19-344.

[56] I. Lopatovska and I. Arapakis, "Theories, Methods and Current Research on Emotions in Library and Information Science", Information Retrieval and Human-Computer Interaction, Information Processing \& Management, vol. 47, no. 4 (2011), pp. 575-592.

[57] D. Bilal and I. Bachir, "Children's Interaction with Cross-Cultural and Multilingual Digital Libraries", International Journal (Information Seeking, Success, and Affective Experience, Information Processing and Management), vol. 42, no. 1, (2007), pp. 65-80.

[58] C. Mooney, M. Scully, G. J. F. Jones and A. F. Smeaton,"Investigating Biometric response for Information Retrieval Applications", Advances in Information Retrieval, Edited M. Lalmas, A. MacFarlane, S. Rüger, A. Tombros, T, Tsikrika and A. Yavlinsky, Springler Berlin Heidelberg (2006), vol. 3936, pp. 570-574.

[59] A. F. Smeaton and S. Rothwell, "Biometric Responses to Music-Rich Segments in Films", The Cdvplex Proceedings of the 2009 Seventh International Workshop on Content-Based Multimedia Indexing, IEEE, (2009) June 3-5; DC, USA.

[60] K.-H. Park and T.-Y. Kim. Facial Color Adaptive Technique Based on the Theory of Emotion-Color Association and Analysis of Animation. The Tenth IEEE International Workshop on Multimedia Signal Processing, (2008) October 8-10. 
[61] O. d. Pos and P. Green-Armytage, "Facial Expressions, Colours and Basic Emotions", Journal of the International Colour Association, vol. 1, no. 1 (2007). pp. 1-20.

[62] M. Argyle, "The Psychology of Interpersonal Behaviour", Penguin UK, (1994).

[63] M. Hinchey, A. Pagnoni, F. J. Rammig and H. Schmeck, "Color Theory and its Application in Art and Design", Springer Publishing Company, Incorporated (2008).

[64] M. H. Alkawaz and A.H. Basori, "The Effect of Emotional Colour on Creating Realistic Expression of Avatar", Proceedings of the 11th ACM SIGGRAPH International Conference on Virtual-Reality Continuum and its Applications in Industry, (2012) December 2-4 ,Singapore.

[65] N. A. Nijdam. "Mapping Emotion to Color", (2009).

[66] C. Donner and H. W. Jensen, "A Spectral BSSRDF for Shading Human Skin", Proceedings of the 17th Eurographics Conference on Rendering Techniques, (2006), pp. 409-417.

[67] C. Donner, T. Weyrich, E. d'Eon, R. Ramamoorthi and S. Rusinkiewicz, "A Layered, Heterogeneous Reflectance Model for Acquiring and Rendering Human Skin", ACM Transactions on Graphics (TOG). vol. 27 , no. $5,(\mathbf{2 0 0 8})$.

[68] M. K. Seo, H. J. Kwon, B. Ahmed, Y. Y. Lee, J. D. Yoo, I. Y. Jang, S. J. Lee, M. k. Park and K. H. Lee, "Rendering of Human Skin During Physical Exercise", ACM SIGGRAPH 2012 Posters, (2012).

[69] F. Pighin, R. Szeliski, and D. H. Salesin, "Resynthesizing Facial Animation Through 3d Model-Based Tracking", The Proceedings of the Seventh IEEE International Conference on Computer Vision, (1999) September 20-27.

[70] A. S. Georghiades, P. N. Belhumeur and D. J. Kriegman, "Illumination-based Image Synthesis: Creating Novel Images of Human Faces Under Differing Pose and Lighting", Proceedings of IEEE Workshop on Multi-View Modeling and Analysis of Visual Scenes, (1999) June 26.

[71] P. Debevec, T. Hawkins, C. Tchou, H.-P. Duiker, W. Sarokin and M. Sagar.. Acquiring the Reflectance Field of a Human Face, Proceedings of the 27th Annual Conference on Computer Graphics and Interactive Techniques, (2000) July 23-28; LA, USA.

[72] T. Hawkins, A. Wenger, C. Tchou, A. Gardner, F. Goransson and P. Debevec.. Animatable Facial Reflectance Fields. Proceedings of the Fifteenth Eurographics conference on Rendering Techniques, (2004); Eurographics Association.

[73] A. Wenger, A. Gardner, C. Tchou, J. Unger, T. Hawkins and P. Debevec, "Performance Relighting and Reflectance Transformation with Time-Multiplexed Illumination", ACM Transactions on Graphics (TOG), vol. 24, no. 3, (2005).

[74] A. Mahmud. Dynamic Facial Appearance Capture Using Six Primaries. Master of Science. The University Of British Columbia. (2012).

[75] A. Jones, A. Gardner, M. Bolas, I. McDowall and P. Debevec, "Performance Geometry Capture for Spatially Varying Relighting", ACM SIGGRAPH 2005 Sketches, (2005) 31 July - 4 August. LA, USA.

[76] P. Hanrahan and W. Krueger, "Reflection from Layered Surfaces due to Subsurface Scattering", Proceedings of the 20th Annual Conference on Computer Graphics and Interactive Techniques, (1993) August 2-6; NY, USA.

[77] J. Stam, "An Illumination Model for a Skin Layer Bounded by Rough Surfaces", Rendering Techniques 2001, Edited S. J. Gotler, and K. Myszkowski, Springer Vienna, (2001), pp. 39-52.

[78] A. Krishnaswamy and G. V. Baranoski, "A Biophysically-Based Spectral Model of Light Interaction with Human Skin", Computer Graphics Forum, Wiley Online Library, vol. 23, no. 3, (2004), pp. 331340.

[79] C. Donner and H. W. Jensen, "Light Diffusion in Multi-Layered Translucent Materials. ACM Transactions on Graphics (TOG), vol. 24, no. 3, (2005), pp. 1032-1039.

[80] H. W. Jensen and J. Buhler, "A Rapid Hierarchical Rendering Technique for Translucent Materials", ACM Transactions on Graphics (TOG), vol. 21, no. 3, (2002), pp. 576-581.

[81] C. Hery, "Implementing a Skin BSSRDF (or several...)", SIGGRAPH 2005 Digital Face Cloning Course Notes, (2005) 31 July - 4 August; LA. USA.

[82] S. Tariq, A. Gardner, I. Llamas, A. Jones, P. Debevec and G. Turk.. Efficient Estimation of Spatially Varying Subsurface Scattering Parameters. Vision, Modeling, and Visualization, (2006); Aachen, Germany.

[83] T. Weyrich, W. Matusik, H. Pfister, B. Bickel, C. Donner, C. Tu, J. McAndless, J. Lee, A. Ngan, H, W. Jensen and M. Gross, "Analysis of Human Faces Using a Measurement-Based Skin Reflectance Model", ACM Transactions on Graphics (TOG), vol. 25, no. 3, (2006). pp. 1013-1024.

[84] E. d'Eon, D. Luebke, and E. Enderton, "Efficient Rendering of Human Ski", Proceedings of the 18th Eurographics Conference on Rendering Techniques, (2007).

[85] M. Goesele, H. P. A. Lensch, J, Lang, C. Fuchs and H.-P. Seidel, "DISCO: Acquisition of Translucent Objects", ACM Transactions on Graphics (TOG), vol. 23, no. 3, (2004), pp. 835-844.

[86] T T. Tong, J. Wang, S. Lin, B. Guo and H.-Y. Shum, "Modeling and Rendering of Quasi-Homogeneous Materials", ACM Transactions on Graphics (TOG), vol. 24, no. 3, (2005), pp. 1054-1061.

[87] P. Peers, K. v. Berge, W. Matusik, R. Ramamoorthi, J. Lawrence, S. Rusinkiewicz and P. Dutré, "A Compact Factored Representation of Heterogeneous Subsurface Scattering", ACM Transactions on Graphics (TOG), vol. 25, no. 3 (2006), pp. 746-753. 
[88] J. Wang, S. Zhao, X. Tong, S. Lin, Z. Lin, Y. Dong and B. Guo and H.-Y. Shum, "Modeling and Rendering of Heterogeneous Translucent Materials Using the Diffusion Equation", Transactions on Graphics (TOG), vol. 27, no. 1, (2008), pp. 9.

[89] A. Ghosh, T. Hawkins, P. Peers, S. Frederiksen and P. Debevec, "Practical Modeling and Acquisition of Layered Facial Reflectance", ACM Transactions on Graphics (TOG), vol.27, no. 5, (2008), pp. 139.

[90] O. G. Cula, K. J. Dana, F. P. Murphy and K. Rao, "Skin Texture Modeling", International Journal of Computer Vision, vol. 62, no. 1-2, (2005), pp. 97-119.

[91] M. Haindl and J. Filip, "Bidirectional Texture Functions", Visual Texture, vol. 29, no. 10, (2013), pp. 147-209.

[92] P. J. Matts, P. K. Dykes, and R. Marks, The Distribution of Melanin in Skin Determined in Vivo. British Journal of Dermatology. 156, 5 (2007). pp. 620-628.

[93] P. G. Cavalcanti, J. Scharcanski, and C. B. O. Lopes, "Shading Attenuation in Human Skin Color Images", Proceedings of the 6th International Conference on Advances in Visual Computing, (2010); Verlag: Las Vegas, NV, USA.

[94] T. Yamada and T. Watanabe, "Virtual Facial Image Synthesis with Facial Color Enhancement and Expression under Emotional Change of Anger", The 16th IEEE International Symposium on Robot and Human Interactive Communication, (2007).

[95] Y. Jung, C. Weber, J. Keil and T. Franke, "Real-Time Rendering of Skin Changes Caused by Emotions", Proceedings of the 9th International Conference on Intelligent Virtual Agents. Edited Z. Ruttkay, M. Kipp, A. Nijholt and H. H. Vilhjálmsson, Springer-Verlag: Amsterdam, Netherlands, (2009), pp. 504-505.

[96] C. M. de Melo and J. Gratch, "Expression of Emotions Using Wrinkles, Blushing, Sweating and Tears. Intelligent Virtual Agents", Edited Z. Ruttkay, M. Kipp, A. Nijholt and H. H. Vilhjálmsson, Springer, (2009), pp. 188-200.

[97] V. Hall. Facial Animation and Speech Synthesis, (1992).

[98] M. M. Khan, R.D. Ward, and M. Ingleby, "Classifying Pretended and Evoked Facial Expressions of Positive and Negative Affective States Using Infrared Measurement of Skin Temperature", vol. 6, no. 1 (2009), pp. 1-22.

[99] F. Ceriani, R. Fogliani, and A. Kustermann, Facial Expressions, "Development of Normal Fetal Movements", Springer Milan, (2010), pp. 77-86.

[100]G. Sandbach, S. Zafeiriou, M. Pantic and L. Yin, "Static and Dynamic 3D Facial Expression Recognition: A Comprehensive Survey", Image Vision Computing, vol. 30, no. 10, (2012), pp. 683-697.

[101]A. Danelakis, T. Theoharis, and I. Pratikakis, "A Survey on Facial Expression Recognition in 3D Video Sequences", Multimedia Tools and Applications, (2014), pp. 1-39.

[102]J. Gorin, M. Raulet, and F. Prêteux, "MPEG Reconfigurable Video Coding: From specification to a reconfigurable implementation",Signal Processing: Image Communicatio, vol. 28, no. 10, (2013). pp. 1224-1238.

[103]T. Ezzat and T. Poggio. MikeTalk: A Talking Facial Display Based on Morphing Visemes. IEEE Conference on Computer Animation, (1998) Jun 8-10. pp. 96-102.

[104]A A. Arya and B. Hamidzadeh, "Personalized Face Animation in Show Face System", Journal of Image and Graphics, Special Issue on Virtual Reality and Virtual Environments, vol. 3, no. 2 (2003), pp. 345363.

\section{Authors}

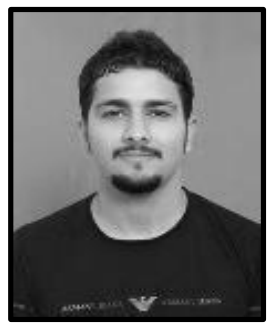

Mohammed Hazim Alkawaz, he was born in Mosul city, Iraq in 1989. He received the B.Sc in computer science from University of Mosul 2010 and M.Sc. degree in computer science from the Universiti Teknologi Malaysia, Johor Bahru, Johor, in 2013. He is a current $\mathrm{Ph}$.D. student in UniversitiTeknologi Malaysia, Johor Bahru, Johor. His research interests include Computer Graphics, Facial Animation and Facial skin color. He has published researches in international journals like IEEE, ACM SIGGRAPH, Springer and has attended international conferences. He also has some papers that are yet to be published. 


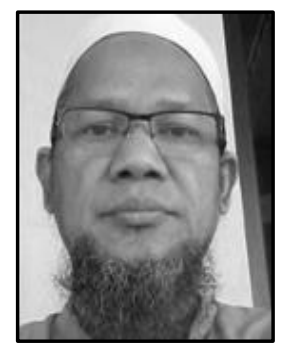

Dzulkifli Mohammad, he is a senior professor in the Faculty of Computing Universiti Teknologi Malaysia (UTM) Johor Malaysia. His keen research interest are facial animation, intelligent features mining, Pattern Recognition and Security. He has supervised several Master and PhD students in the field of Computer Science and Information Security. He is author of more than 300 publications that are published in world class conferences and journals of international repute.

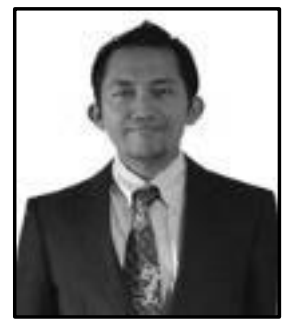

Ahmad Hoirul Basori, he received the B.Sc(Software Engineering) in 2004 from Institut Teknologi Sepuluh Nopember Surabaya, Indonesia. and the Ph.D. degree in Computer Graphics from Universiti Teknologi Malaysia, Johor Bahru, Johor, in 2011. From 2004 to present, he is a lecturer with the Department of Informatics, Faculty of Information Technology, Institut Teknologi Sepuluh Nopember Surabaya, Indonesia. Since 2011, he join Department of Computer Graphics and Multimedia, Universiti Teknologi Malaysia as Assistant Professor. He alsoappointed aseditorial board and reviewer of some international journal and conferences. Authors ofmore than 60 articles in referred journal and international conferences, member of professional membership IEEE, ACM SIGGRAPH, IAENG and Senior Member of IACSIT. His research interests include Computer Graphics, Facial Animation, Cloth Simulation, Medical Visualization, Haptic Interaction and Man Machine Interaction.

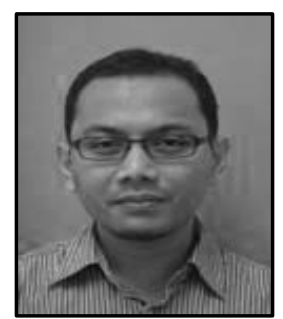

Farhan Mohamed, he is a lecturer at the Faculty of Computing and Research Fellow at Media and Game Innovation Centre of Excellence (MAGIC-X) at Universiti Teknologi Malaysia. His PhD research was conducted at the Swansea University. His research explored the possibilities of using visual metaphors and spatiality of human perceptions for tangible and abstract data in a visualization and virtual 3-Dimensional space. The work was a result of collaboration of UTM, Oxford University, and Swansea University. He has received a Bachelor of Science degree in Computer Science from Universiti Teknologi Malaysia with an Excellence Student Award by Juita-UTM in 2003. 
International Journal of Multimedia and Ubiquitous Engineering Vol. 10, No. 1 (2015) 